\title{
Some New Strongly Almost Summable Difference Sequence Spaces
}

\author{
Zakawat U. Siddiqui ${ }^{1}$, Abayomi O. Mosaku ${ }^{1} \&$ Ibrahim M. Dibal ${ }^{2}$ \\ ${ }^{I}$ Department of Mathematics and Statistics, University of Maiduguri, Maiduguri, Borno State \\ ${ }^{2}$ Department of Mathematics and Statistics, Federal Polytechnic, Damaturu, Yobe State
}

\begin{abstract}
Sequences are indispensible to the pure and applied mathematicians, and thus, by extension, to all field of sciences. The idea of difference sequence spaces was introduced in 1981, which has been generalized. The space of almost convergence sequences introduced in 1948 was generalized in 1978. Almost convergence difference sequence spaces have undergone series of gradual generalization. The purpose of this paper is to introduce the concept of $\Delta_{v^{\lambda}}^{m}$ almost strongly summable difference sequence spaces with respect to a sequence of moduli on a seminorm $q$ and to examine some properties of the three introduced sequence spaces which generalize many existing results, Various topological properties of these spaces with some of their inclusion relations, were studied including their solidity, symmetricity, sequence algebra, convergence free and so on. In this work, concerted effort has been made to generalize some existing sequence spaces by extending their definitions to a wider space in which the existing ones are contained.
\end{abstract}

Key Words: Difference Sequence Spaces, Strongly Almost summable sequences, Modulus Function.

\section{Introduction}

A sequence space is a linear space whose elements are infinite sequences of real or complex numbers. Equivalently, it is a function space whose elements are functions from the natural number to the field $\mathrm{K}$ of real or complex numbers.

Let $\omega$ denote the space of all sequences of complex numbers. The spaces $l_{\infty}, c$ and $c_{0}$ are of particular interest in this paper which are defined as the linear spaces of bounded, convergent and null sequences $x=\left(x_{k}\right)_{k=0}^{\infty}$, respectively, such that $c_{0} \subset c \subset l_{\infty}$ and normed by

$$
\square x \square_{\infty}=\operatorname{Sup}_{k}\left|x_{k}\right|, \forall k \in \square
$$

and are expressed as follows:

$$
\begin{gathered}
l_{\infty}=\left\{x=\left(x_{k}\right) \in \omega: \operatorname{Sup}_{k}\left|x_{k}\right|<\infty\right\} \\
c=\left\{x=\left(x_{k}\right) \in \omega: \lim _{k \rightarrow \infty} x_{k}=l \text { for some } l \in \square\right\} \\
c_{0}=\left\{x=\left(x_{k}\right) \in \omega: \lim _{k \rightarrow \infty} x_{k}=0\right\}
\end{gathered}
$$

It is not difficult to see that $\ell_{\infty}, c$ and $c_{0}$ are normed linear spaces and that $l_{\infty}$ is a Banach space. Kizmaz [1] introduced the notion of difference sequence spaces as follows:

$$
X(\Delta)=\left\{x=\left(x_{k}\right) \in \omega:\left(\Delta x_{k}\right) \in X\right\}
$$

where $X=l_{\infty}, c$ and $c_{0}$ and

They are Banach spaces with the norm

$$
\Delta x=\Delta x_{k}=\left(x_{k}-x_{k+1}\right)
$$

$$
\begin{array}{r}
\|x\|_{\Delta}=\left|x_{1}\right|+\|\Delta x\|_{\infty} \\
\Rightarrow\|x\|_{\Delta}=\left|x_{1}\right|+\sup _{k}\left|\Delta x_{k}\right|
\end{array}
$$

And later on this notion was generalized as follows 


$$
X\left(\Delta^{m}\right)=\left\{x=\left(x_{k}\right) \in \omega:\left(\Delta^{m} x_{k}\right) \in X\right\}
$$

for $X=l_{\infty}, c$ and $c_{0}$, where $m \in \square$, where

such that

$$
\begin{aligned}
& \Delta^{m} x=\Delta^{m} x_{k}=\left(\Delta^{m-1} x_{k}-\Delta^{m-1} x_{k+1}\right) \\
& \Delta x_{k}=\left(x_{k}-x_{k+1}\right) \\
& \text { and } \Delta^{0} x=\left(x_{k}\right) .
\end{aligned}
$$

$$
\Delta^{m} x_{k}=\sum_{i=0}^{m}(-1)^{i}\left(\begin{array}{c}
m \\
i
\end{array}\right) x_{k+i}
$$

They are Banach spaces with norm defined by

$$
\begin{gathered}
\square x \square_{\Delta^{m}}=\sum_{i=1}^{m}\left|x_{i}\right|+\square \Delta^{m} x \square_{\infty} \\
\Rightarrow \square x \square_{\Delta^{m}}=\sum_{i=1}^{m}\left|x_{i}\right|+\sup _{k}\left|\Delta^{m} x_{k}\right|
\end{gathered}
$$

Worthy it is to mention that recently, the sequence spaces $X\left(\Delta^{m}\right)$ were generalized by Et and Esi [3] to the following sequence space

$$
X\left(\Delta_{v}^{m}\right)=\left\{x=\left(x_{k}\right) \in \omega:\left(\Delta_{v}^{m} x_{k}\right) \in X\right\}
$$

for $X=l_{\infty}, c$ and $c_{0}$, where

$$
\Delta_{v}^{0} x=\left(v_{k} x_{k}\right), \Delta_{v} x_{k}=\left(v_{k} x_{k}-v_{k+1} x_{k+1}\right)
$$

and

such that

$$
\Delta_{v}^{m} x=\Delta_{v}^{m} x_{k}=\left(\Delta_{v}^{m-1} x_{k}-\Delta_{v}^{m-1} x_{k+1}\right)
$$

$$
\Delta_{v}^{m} x_{k}=\sum_{i=0}^{m}(-1)^{i}\left(\begin{array}{c}
m \\
i
\end{array}\right) v_{k+1} x_{k+i}
$$

and $v=\left(v_{k}\right) \neq 0$ is any fixed sequence of non-zero numbers for all $k \in \square$. (see [3]) we claim that the generalized sequence space

$$
X\left(\Delta_{v}^{m}\right) \text { where } X=l_{\infty}, c \text { or } c_{0}
$$

Is a Banach space with norm defined by

$$
\|x\|_{\Delta_{v}^{m}}=\sum_{i=1}^{m}\left|x_{i}\right|+\sup \left|\Delta_{v}^{m} x_{k}\right|
$$

Subsequently, difference sequence spaces have been studied by Asma and Et [4], Bektas et al [5], Colak ([6] and [7]), Isik [8] and Mursaleen [9].

There is an important notion (modulus function) used in this paper which was introduce by Nakano [10]. Now we give the definition of the modulus function.

Definition: A function $f:[0, \infty) \rightarrow[0, \infty)$ is called a modulus function if

1. $f(t)=0$ if and only if $t=0$

2. $f(t+u) \leq f(t)+f(u)$ for all $t, u \geq 0$

3. $f$ is increasing and

4. $\quad f$ is continuous from the right of 0 .

Because of (2), $|f(x)-f(y)| \leq f(x-y)$

So that in view of $(4) \mathrm{f}$ is continuous on $[0, \infty)$ 
A modulus may be bounded or unbounded. For example, $f(x)=x^{p},(0<p \leq 1)$ is unbounded and $f(x)=\frac{x}{1+x}$ is bounded .

Let $X$ be a sequence space. For a modulus function $f$, the sequence space $X(f)$ is defined as

$$
X(f)=\left\{x=\left(x_{k}\right) \in \omega:\left(f\left(\left|x_{k}\right|\right)\right) \in X\right\}
$$

An extension of $X(f)$ is obtained by considering a sequence of moduli $F=\left(f_{k}\right)$.

$$
X(F)=\left\{x=\left(x_{k}\right)_{k=1}^{\infty} \in \omega:\left(f_{k}\left(\left|x_{k}\right|\right)\right) \in X\right\},
$$

where $X=l_{\infty}, c$ and $\mathrm{c}_{0}$. (see [11])

Later, Gaur and Mursaleen [12] defined the following sequence spaces:

$$
\begin{aligned}
& l_{\infty}(F, \Delta)=\left\{x=\left(x_{k}\right) \in \omega:\left(x_{k}\right) \in l_{\infty}(F)\right\} \\
& c_{0}(F, \Delta)=\left\{x=\left(x_{k}\right) \in \omega:\left(x_{k}\right) \in c_{0}(F)\right\} \\
& c(F, \Delta)=\left\{x=\left(x_{k}\right) \in \omega:\left(x_{k}\right) \in c(F)\right\}
\end{aligned}
$$

Recently Khan [13] defined the following sequence spaces

In particular;

$$
X(F, p)=\left\{x=\left(x_{k}\right)_{k=1}^{\infty} \in \omega:\left(f_{k}\left(\left|x_{k}\right|^{p_{k}}\right)\right) \in X(p)\right\}
$$

$$
\begin{gathered}
l_{\infty}(F, p)=\left\{x=\left(x_{k}\right)_{k=1}^{\infty} \in \omega: \sup _{k} f_{k}\left|x_{k}\right|^{p_{k}}<\infty\right\} \\
c_{0}(F, p)=\left\{x=\left(x_{k}\right)_{k=1}^{\infty} \in \omega: f_{k}\left(\left|x_{k}\right|^{p_{k}}\right) \rightarrow 0 ;(k \rightarrow \infty)\right\}, \\
c(F, p)=\left\{x=\left(x_{k}\right)_{k=1}^{\infty} \in \omega: f_{k}\left(\left|x_{k}\right|^{p_{k}}\right) \rightarrow l,(k \rightarrow \infty)\right\},
\end{gathered}
$$

and

$$
\begin{aligned}
& l_{\infty}\left(F, p, \Delta^{m}\right)=\left\{x=\left(x_{k}\right)_{k=1}^{\infty} \in \omega:\left(\Delta^{m} x_{k}\right) \in l_{\infty}(F, p)\right\}, \\
& c_{0}\left(F, p, \Delta^{m}\right)=\left\{x=\left(x_{k}\right)_{k=1}^{\infty} \in \omega:\left(\Delta^{m} x_{k}\right) \in c_{0}(F, p)\right\} . \\
& c\left(F, p, \Delta^{m}\right)=\left\{x=\left(x_{k}\right)_{k=1}^{\infty} \in \omega:\left(\Delta^{m} x_{k}\right) \in c(F, p)\right\} .
\end{aligned}
$$

for any sequence of moduli $F=\left(f_{k}\right)$.

The concept of almost convergence was defined by Lorentz in 1948, using the idea of Banach limits. A sequence $x=\left(x_{k}\right) \in l_{\infty}$ is said to be almost convergent to the generalized limit $\alpha$ if all Banach limits of $\mathrm{x}$ are coincide and are equal to $\alpha$, this is denoted by $f-\lim x_{k}=\alpha$. (see Lorentz [14]) proved that $f-\lim x_{k}=\alpha$ if and only if $\lim _{n} \frac{1}{n+1} \sum_{k=1}^{n} x_{k+s}=\alpha$ uniformly in s. In the case $\alpha=0$, the sequence $\mathrm{x}$ is called almost null. The spaces of almost convergent and null sequences are denoted by $\hat{c}$ and $\hat{c}_{0}$ respectively. It is well-known that a convergent sequence is almost convergent such that its ordinary and generalized limits are equal.

The strong almost convergence of a complex sequence $\mathrm{x}$ to number 1 was defined by

$$
\frac{1}{n} \sum_{k=0}^{n}\left|x_{k+s}-l\right| \rightarrow 0 \text {, as } m \rightarrow \infty \text {, uniformly is } s, \text { (see [15] and [16]) }
$$


which leads to the concept of strong almost convergence sequences as $[\hat{c}]$, we denote the spaces of all strongly almost convergence sequence. It is immediate that the inclusion $[\hat{c}] \subset \hat{c}$ strictly holds. Also $[\hat{c}]$ is closed subspace of $c \subset[\hat{c}] \subset \hat{c} \subset l_{\infty}$ strictly hold.

A sequence $x=\left(x_{k}\right)$ is said to be summable $(c, 1)$ or strongly summable $[c, 1]$ if and only if $\lim _{n} \frac{1}{n} \sum_{k 1}^{n} x_{k}$ exists.

Spaces of strongly Cesáro summable sequences were discussed by Kuttner in 1946 [17] and this concept was generalized by Maddox (see [16]) and some others. The class of sequences which are strongly Cesáro summable with respect to a modulus was introduces by Maddox (see 15]) as an extension of the concept of the strong Cesáro summable.

A sequence $x=\left(x_{k}\right)$ is said to be $(v, \lambda)$-summable to a number 1 if $t_{n}(x) \rightarrow l$ as $n \rightarrow \infty$. We write

$$
\begin{gathered}
{[V, \lambda]_{0}=\left\{x=\left(x_{k}\right) \in \omega: \lim _{n} \frac{1}{\lambda_{n}} \sum_{k \in I_{n}}\left|x_{k}\right|=0\right\}} \\
{[V, \lambda]=\left\{x=\left(x_{k}\right) \in \omega: \lim _{n} \frac{1}{\lambda_{n}} \sum_{k \in I_{n}}\left|x_{k}-l\right|=0, \text { for some } l\right\} \text { and }} \\
{[V, \lambda]_{\infty}=\left\{x=\left(x_{k}\right) \in \omega: \sup _{n} \frac{1}{\lambda_{n}} \sum_{k \in I_{n}}\left|x_{k}\right|<\infty\right\}}
\end{gathered}
$$

Since $\lambda=\left(\lambda_{n}\right)$ is a non decreasing sequence of positive reals tending to infinity, but $\lambda_{1}=1$ and $\lambda_{n+1} \leq \lambda_{n}+1$. The generalized de la Vallee-Poussin means is defined as

where

$$
t_{n}(x)=\frac{1}{\lambda_{n}} \sum_{k \in I_{n}} x_{k}
$$

$$
I_{n}=\left[n-\lambda_{n}+1, n\right]
$$

The following inequality would be used throughout this paper

Let $P=\left(p_{k}\right)$ be a sequence of positive real number with

$$
0<p_{k} \leq \operatorname{Sup}_{k} p_{k}=H<\infty, D=\max \left(1,2^{H-1}\right) .
$$

Then, for $a_{k}, b_{k} \in \square$, we have.

$$
\left|a_{k}+b_{k}\right|^{p_{k}} \leq D\left\{\left|a_{k}\right|^{p_{k}}+\left|b_{k}\right|^{p_{k}}\right\}
$$

Furthermore, Let $X, Y \subset \omega$, then we shall write

$$
M(X, Y)=\bigcap_{x \in X} x^{-1} * Y=\{a \in \omega: a x \in Y \text { for all } x \in X\},
$$

the set $X^{\alpha}=M\left(X, l_{1}\right)$ is called the Kothe-Toeplitz dual space or $\alpha$-dual of $X$. A sequence space $\mathrm{X}$ is said to be:

i.) Solid (or normal) if for all the sequences of scalar $\left(\alpha_{k}\right)$ with $\left|\alpha_{k}\right| \leq 1, \forall k \in \square$ then $\left(\alpha_{k} x_{k}\right) \in X$ whenever $\left(x_{k}\right) \in X$

ii.) $\quad$ Symmetric if $\left(x_{k}\right) \in X$ implies $\left(x_{\pi(k)}\right) \in X$, where $\pi(k)$ is a permutation of $\square$. 
iii.) A sequence Algebra if $\left(x_{k}\right),\left(y_{k}\right) \in X$ implies $\left(x_{k} y_{k}\right) \in X$.

iv.) Convergence free if $\left(y_{k}\right) \in X$ whenever $\left(x_{k}\right) \in X$ and $y_{k}=\theta$ whenever $x_{k}=\theta$

For any two seminorms $\mathrm{p}$ and $\mathrm{q}, \mathrm{p}$ is said to be stronger than $\mathrm{q}$ if whenever $\left(x_{k}\right)$ is a sequence such that $p\left(x_{k}\right) \rightarrow 0$ as $k \rightarrow \infty$, then also $q\left(x_{k}\right) \rightarrow 0$ as $k \rightarrow \infty$. If $\mathrm{p}$ is stronger than $\mathrm{q}$ then, $\mathrm{q}$ is said to be weaker than $\mathrm{p}$. If $\mathrm{p}$ is stronger than $\mathrm{q}$ and $\mathrm{q}$ is stronger than $\mathrm{p}$, then $\mathrm{p}$ and $\mathrm{q}$ are called equivalent.

Let $F=\left(f_{k}\right)$ be any sequence of moduli, $p=\left(p_{k}\right)$ be a sequence of strictly positive real numbers and $v=\left(v_{k}\right)$ be any fixed sequence of non zero complex number and $u=\left(u_{k}\right) \neq 0$ be any sequence of all $\mathrm{k}$ with, a seminorn q. We defined the following sequence space as:

$$
\begin{gathered}
{\left[\hat{V}, \Delta_{v^{\lambda}}^{m}, F, p, q, u\right]_{0}=\left\{x \in \omega: \lim _{n} \frac{1}{\lambda_{n}} \sum_{k \in I_{n}} u_{k}\left[f_{k}\left(\left|q\left(\Delta_{v}^{m} x_{k+j}\right)\right|\right)\right]^{p_{k}}=0\right\},} \\
{\left[\hat{V}, \Delta_{v^{\lambda}}^{m}, F, p, q, u\right]_{1}=\left\{x \in \omega: \lim _{n} \frac{1}{\lambda_{n}} \sum_{k \in I_{n}} u_{k}\left[f_{k}\left(\left|q\left(\Delta_{v}^{m} x_{k+j}-l\right)\right|\right)\right]^{p_{k}}=0, \text { for some } l\right\} .}
\end{gathered}
$$

And,

$$
\left[\hat{V}, \Delta_{v^{\lambda}}^{m}, F, p, q, u\right]_{\infty}=\left\{x \in \omega: \sup _{n} \frac{1}{\lambda_{n}} \sum_{k \in I_{n}} u_{k}\left[f_{k}\left(\left|q\left(\Delta_{v}^{m} x_{k+j}\right)\right|\right)\right]^{p_{k}}<\infty\right\}
$$

uniformly in $\mathrm{j}$.

Throughout this paper we shall make $\mathrm{z}$ to denote any of the notion 0,1 and $\infty$.

If $q(x)=|x|, u_{k}=1$ and $v=(1,1,1, \ldots)$ then

$$
\left[\hat{V}, \Delta_{v^{\lambda}}^{m}, F, p, q, u\right]_{z}=\left[\hat{V}_{\lambda}, \Delta^{m}, F, p\right]_{z} \quad(\text { see }[19])
$$

If $q(x)=|x|, u_{k}=1$ then

$$
\left[\hat{V}, \Delta_{v^{\lambda}}^{m}, F, p, q, u\right]_{z}=\left[\hat{V}, \Delta_{v^{\lambda}}^{m}, F, p\right]_{z}
$$

If $q(x)=|x|, u_{k}=1$ and $\lambda_{n}=n$ then

$$
\left[\hat{V}, \Delta_{v^{\lambda}}^{m}, F, p, q, u\right]_{z}=\left[\hat{V}, \Delta_{v}^{m}, F, p\right]_{z} \quad(\text { see }[20])
$$

$q(x)=|x|, u_{k}=1, m=0, f_{k}(x)=x, \lambda_{n}=n$ and $v=(1,1,1, \ldots) \forall k \geq 0$ then

$$
\left[\hat{V}, \Delta_{v^{\lambda}}^{m}, F, p, q, u\right]_{z}=[\hat{V}, p]_{z} \quad(\text { see Maddox }[15])
$$

$q(x)=|x|, u_{k}=1, m=0, f_{k}(x)=x, \lambda_{n}=n, p_{k}=1$ and $v=(1,1,1, \ldots) \forall k \geq 0$ then

$$
\left[\hat{V}, \Delta_{v^{\lambda}}^{m}, F, p, q, u\right]_{z}=[\hat{V}]_{z} \quad(\text { see }[19])
$$

We prove the following results.

\section{Main Results}

Theorem 2.1: Suppose $p=\left(p_{k}\right)$ be bounded. Then the spaces $\left[\hat{V}, \Delta_{v^{\nu}}^{m}, F, p, q, u\right]_{z}$ are linear spaces over the set of complex $\square$ where $z=1,0$ and $\infty$

Proof:

We shall give the proof for $\left[\hat{V}, \Delta_{v^{\lambda}}^{m}, F, p, q, u\right]_{0}$ only, the other can be treated similarly. Let $x, y \in\left[\hat{V}, \Delta_{v^{\lambda}}^{m}, F, p, q, u\right]_{0}$, for $\alpha, \beta \in \square$ their exist positive integers $M_{\alpha}$ and $N_{\beta}$ such that $|\alpha| \leq M_{\alpha}$ and $|\beta| \leq N_{\beta}$ since $f_{k}$ is subadditive, $\mathrm{q}$ is a seminorm and $\Delta_{v}^{m}$ is linear 


$$
\begin{aligned}
\lim _{n} & \frac{1}{\lambda_{n}} \sum_{k \in I_{n}} u_{k}\left[f_{k}\left(\left|q\left(\Delta_{v}^{m}\left(\alpha x_{k+j}+\beta y_{k+j}\right)\right)\right|\right)\right]^{p_{k}} \\
& \leq D\left(\max \left(1,\left|M_{\alpha}\right|^{H}\right)\right) \lim _{n} \frac{1}{\lambda_{n}} \sum_{k \in I_{n}} u_{k}\left[f_{k}\left(\left|q\left(\Delta_{v}^{m}\left(x_{k+j}\right)\right)\right|\right)\right]^{p_{k}} \\
& +D\left(\max \left(1,\left|N_{\beta}\right|^{H}\right)\right) \lim _{n} \frac{1}{\lambda_{n}} \sum_{k \in I_{n}} u_{k}\left[f_{k}\left(\left|q\left(\Delta_{v}^{m}\left(y_{k+j}\right) \mid\right)\right|\right)\right]^{p_{k}}
\end{aligned}
$$

Hence, $\left[\hat{V}, \Delta_{v^{\lambda}}^{m}, F, p, q, u\right]_{z}$ is a linear space.

Theorem 2.2: The sequence spaces

$\left[\hat{V}, \Delta_{v^{\lambda}}^{m}, F, p, q, u\right]_{0},\left[\hat{V}, \Delta_{v^{\lambda}}^{m}, F, p, q, u\right]_{1}$ and $\left[\hat{V}, \Delta_{v^{\lambda}}^{m}, F, p, q, u\right]_{\infty}$ are not normal for $m \geq 1$

Proof:

Let $q(x)=|x|, u_{k}=1, f_{k}(x)=x, \lambda_{n}=n$ for all $n \in \square, p=\left(p_{k}\right)=1$ and $v=(1,1,1, \ldots), \forall k \geq 0$, then $\left(x_{k}\right)=\left(k^{m}\right) \in\left[\hat{V}, \Delta_{v^{\lambda}}^{m}, F, p, q, u\right]_{\infty}$ but $\left(\alpha_{k} x_{k}\right) \notin\left[\hat{V}, \Delta_{v^{\lambda}}^{m}, F, p, q, u\right]_{\infty}$, when $\alpha_{k}=(-1)^{k}$ for all $k \in \square$ hence $\left[\hat{V}, \Delta_{v^{2}}^{m}, F, p, q, u\right]_{\infty}$ is not solid. The other cases can be poved by considering similar example

Theorem 2.3: The sequence spaces

$$
\left[\hat{V}, \Delta_{v^{\lambda}}^{m}, F, p, q, u\right]_{0},\left[\hat{V}, \Delta_{v^{\lambda}}^{m}, F, p, q, u\right]_{1} \text { and }\left[\hat{V}, \Delta_{v^{\lambda}}^{m}, F, p, q, u\right]_{\infty}
$$

are not symmetric for $m \geq 1$

Proof:

Let $q(x)=|x|, u_{k}=1, f_{k}(x)=x, \lambda_{n}=n$ for all $n \in \square, p=\left(p_{k}\right)=1$ and $v=(1,1,1, \ldots), \forall k \geq 0$ then $\left(x_{k}\right)=\left(k^{m}\right) \in\left[\hat{V}, \Delta_{v^{\lambda}}^{m}, F, p, q, u\right]_{\infty}$, let $\left(y_{k}\right)$ be an arrangement of $\left(x_{k}\right)$ which is defined by

$$
\left(y_{k}\right)=\left\{x_{1}, x_{2}, x_{4}, x_{3}, x_{9}, x_{5}, x_{16}, x_{25}, x_{7}, x_{36}, x_{10}, x_{49}, x_{8}, \ldots\right\} \text { then } y \notin\left[\hat{V}, \Delta_{v^{\lambda}}^{m}, F, p, q, u\right]_{\infty} \text {. }
$$

Theorem 2.4: For any two sequences $p=\left(p_{k}\right)$ and $t=\left(t_{k}\right)$ of strictly positive real numbers and for any two seminorms, we have $\left[\hat{V}, \Delta_{v^{\lambda}}^{m}, F, p, q_{1}, u\right]_{z} \cap\left[\hat{V}, \Delta_{v^{\lambda}}^{m}, F, t, q_{2}, u\right]_{z} \neq \varnothing \quad$ for $z=0,1$ and $\infty$

Proof:

Since the zero element belongs to each of the above classes of sequences, the intersection is nonempty.

Theorem 2.5: The sequence spaces $\left[\hat{V}, \Delta_{v^{\lambda}}^{m}, F, p, q_{1}, u\right]_{z}$ are not convergence free

Proof: Let $f_{k}(x)=x$, for all $x \in[0, \infty), m=1$ and $v=\left(v_{k}\right)=(1,1,1, \ldots)$ for all $k \in \square$. Let $\mathrm{p}_{\mathrm{k}=1}$ for $\mathrm{k}$ odd $\mathrm{p}_{\mathrm{k}=1}$ for $\mathrm{k}$ even. Consider the sequence $\left(x_{k}\right)$ defined as $x_{k}=k^{-1}$, for all $k \in \square$. Then $\left(x_{k}\right) \in[\hat{V}, \Delta, p]_{z}$ for $z=0,1$ or $\infty$. Consider the sequence $\left(y_{k}\right)$ defined as $y_{k}=k^{2}$, for all $k \in \square$. Then $\left(y_{k}\right)$ neither belong to $[\hat{V}, \Delta, p]_{1}$ nor to $[\hat{V}, \Delta, p]_{\infty}$. Hence, the spaces are not convergence free.

Theorem 2.6: Let $F=\left(f_{k}\right)$ be sequence of moduli, then i.) $\left[\hat{V}, \Delta_{v^{\lambda}}^{m}, F, p, q, u\right]_{0} \subseteq\left[\hat{V}, \Delta_{v^{\lambda}}^{m}, F, p, q, u\right]_{\infty}$ ii.) $\left[\hat{V}, \Delta_{v^{\lambda}}^{m}, F, p, q, u\right]_{1} \subseteq\left[\hat{V}, \Delta_{v^{\lambda}}^{m}, F, p, q, u\right]_{\infty}$ and the inclusion are strict.

Proof: We shall only prove the second inclusion, while the first is obvious. 
Let $x \in\left[\hat{V}, \Delta_{v^{\lambda}}^{m}, F, p, q, u\right]$, by definition of modulus function and inequality 1 , we have

$$
\frac{1}{\lambda_{n}} \sum_{k \in I_{n}} u_{k}\left[f_{k}\left(\left|q\left(\Delta_{v}^{m} x_{k+j}\right)\right|\right)\right]^{p_{k}} \leq D \frac{1}{\lambda_{n}} \sum_{k \in I_{n}} u_{k}\left[f_{k}\left(\left|q\left(\Delta_{v}^{m} x_{k+j}-l\right)\right|\right)\right]^{p_{k}}+D \frac{1}{\lambda_{n}} \sum_{k \in I_{n}} u_{k}\left[f_{k}(|q(l)|)\right]^{p_{k}}
$$

Then, there exist an integer $k_{l}$ such that $q(l) \leq k_{l}$, hence we have

$$
\frac{1}{\lambda_{n}} \sum_{k \in I_{n}} u_{k}\left[f_{k}\left(\left|q\left(\Delta_{v}^{m} x_{k+j}\right)\right|\right)\right]^{p_{k}} \leq D \frac{1}{\lambda_{n}} \sum_{k \in I_{n}} u_{k}\left[f_{k}\left(\left|q\left(\Delta_{v}^{m} x_{k+j}-l\right)\right|\right)\right]^{p_{k}}+\max \left[1,\left(\left(k_{l}\right) f(1)\right)^{H}\right]
$$

Therefore, $x \in\left[\hat{V}, \Delta_{v^{\lambda}}^{m}, F, p, q, u\right]_{\infty}$

We now show that the inclusions are strict.

Let $q(x)=|x|, f_{k}=x, u_{k}=0, v=\left(v_{k}\right)=(1,1,1, \ldots)$ and $p_{k}=1$ for all $k \in \square$. Then $x=\left(k^{m}\right) \in\left[\hat{V}, \Delta_{v^{\lambda}}^{m}, F, p, q, u\right]_{\infty}$, but $x \notin\left[\hat{V}, \Delta_{v^{\lambda}}^{m}, F, p, q, u\right]_{0}$ since $\Delta_{v}^{m}\left(k^{m}\right)=(-1)^{m} m !$.

Under the above restrictions on $\mathrm{q}, \mathrm{F}, \mathrm{u}, \mathrm{v}$ and $\mathrm{p}$. Consider the sequence $x=\left((-1)^{k}\right)$, then $x \in\left[\hat{V}, \Delta_{v^{\lambda}}^{m}, F, p, q, u\right]_{\infty}$ but $x \notin\left[\hat{V}, \Delta_{v^{\lambda}}^{m}, F, p, q, u\right]_{0}$.

\section{Conclusion}

It is necessary to note that sequences are of significant importance in the field of mathematics and by extension, to sciences and beyond. The sequential arrangement of the functionary parts of some machines including constant applications in some aspect of human endeavours, such as the sequential order of the genetic made-up in all living organisms whose alteration can result into deformity (technically called mutations in biology) and others, were some of the motivating factors that necessitated the study of various sequences. In this paper, deliberate attempt was made to construct new sequence spaces so as to generalize the old sequence spaces by extending their definitions. Some properties of the defined spaces were also studied.

\section{References}

[1]. Kızmaz, H. (1981), On certain sequence spaces, Canada Math Bull, 24 (2): 169-176.

[2]. Et, M, and Çolak, R. (1995), On generalized difference sequence spaces, Soochow J Math, 21 (4): 377 386.

[3]. Et, M. and Esi, A. (2000), On Köthe-Toeplitz duals of generalized difference sequence spaces, Bull Malaysian Math Sci. Soc, 23 (1): 25-32.

[4]. Asma, C.and Et M. (1998), On v-invariant sequence spaces. Soochow J Math, 24 (4): 305-308.

[5]. Bektas, C. A., Et M and Colak R. (2004), Generalized difference sequence spaces and their dual spaces, J Math Anal Appl, 292 (2): 423-432.

[6]. Çolak, R. (1989), On invariant sequence spaces, Erciyes Univ Journal of Sci, $5: 881-888$.

[7]. Çolak, R. (1989), On some generalized sequence spaces, Comm Fac Sci Univ Ankara Ser A1 Math Statist, 38(1-2): 35-46.

[8]. Isik, M. (2004), On statistical convergence of generalized difference sequences, Soochow J Math, 30 (2) : 197-205.

[9]. Mursaleen, M. (1996), Generalized spaces of difference sequences, J Math Anal Appl, 203 (3) : 738-745.

[10]. Nakano, H. (1953), Modular sequence spaces, Proc. Japan Acad, 27 : 508-512.

[11]. Kolk, E. (1994), On strong boundedness and summmability with respect to a sequence of moduli, Acta Comment. Univ. Tartu, $960: 41-50$.

[12]. Gaur A. K, Mursaleen (1998), Difference sequence spaces defined by a sequence of moduli, Demonstration Math, 31: 275-278.

[13]. Khan V. A. (2011), Spaces of strongly almost summable difference sequences, Acta Universitatis Apulensis. 28: 261-270.

[14]. Lorentz, G.G., (1948). A contribution to the theory of divergent sequences. Acta Mathematica, 80 (1) : $167-190$.

[15]. Maddox, I. J. (1978), Sequence spaces defined by a modulus, Math Proc Camb. Philos. Soc, 100:161166.

[16]. Maddox I.J. (1986), Sequence spaces defined by a modulus, Mat. Proc. Camb. Phil. Soc. 100, 161-166.

[17]. Kuttner, B. (1946), Note on strong summability, J. London Math. Soc. 40, $118-22$. 
[18]. Maddox, I. J. (1967), Spaces of strongly summable sequences, Quart J Math Oxford, 2 (18) : 345-355.

[19]. Aiyub, M. (2013), Strongly almost summable difference sequences and statistical convergence, Advances in Math. Scientific Journal 2, no. 1, 1-8.

[20]. Khan V. A. and A. Ahmad (2011): Some new generalized difference sequence spaces defined by a sequence of moduli, Appl. Math. J. Chinese Univ. 26(1): 104-108. 